\title{
Biofilms: structures that may facilitate cell-cell interactions
}

\author{
Jonathan P Remis, J William Costerton and Manfred Auer
}

The ISME Journal (2010) 4, 1085-1087; doi:10.1038/

ismej.2010.105; published online 15 July 2010

The vast majority of bacteria live in highly organized communities called biofilms that are usually associated with surfaces or with interfaces. Compared with their planktonic lifestyle, the physiological behavior of biofilm community members is profoundly altered, resulting in an increased resistance to a wide variety of environmental stresses, including antibiotics, desiccation, predation and host immune response (Stoodley et al., 2002). Because the biofilm responds as a community rather than as an assemblage of individual cells, cell-to-cell coordination and therefore communication and signaling are imperative. Quorum sensing, the secretion and detection of a diffusible molecule by the community members, can lead microbial species to suddenly change their behavior, for example, the production of virulence factors (Fuqua and Greenberg, 2002). Beveridge and colleagues for decades have advocated for membrane vesicles as an important but overlooked component of the matrices of biofilms (Schooling and Beveridge, 2006), and more recently have suggested that membrane vesicles may have an important role for intercellular DNA transfer (Schooling et al., 2009). Mashburn and Whiteley (2005) have invoked membrane vesicles as a mechanism for the direct cell-to-cell transmission of a certain quorum-sensing signal that has been implicated in the initiation and control of biofilm formation (Davies et al., 1998).

To study the organization of bacterial biofilms at macromolecular resolution, we resorted to Myxococcus xanthus, a poster child for complex social behavior and biofilm formation. The key for meaningful ultrastructural studies was the faithful preservation of the biofilm using high-pressure freezing and freeze-substitution, which overcomes artifacts typically associated with classical chemical fixation, and room-temperature sample preparation protocols (reviewed in McDonald and Auer, 2006).

With such faithful preservation it became apparent that the abundance of extracellular vesicle structures, found at unprecedented levels, needed to be further examined by electron tomography three-dimensional (3D) ultrastructural imaging.

These structural data are presented in our recent paper in the Journal of Bacteriology (Palsdottir et al.,
2009), with a full description of the manner in which biofilms were grown, and a full description of the sophisticated preparative and 3D imaging technologies. Electron tomography of biofilms formed by cells of $M$. xanthus growing on a cellulose substratum shows that the spaces between these cells are virtually full of vesicles that range in size between 30 and $60 \mathrm{~nm}$ (Figure 1a). Because our preparative methods maintain true volumes, without shrinkage, we can assume that the number and distribution of the intercellular vesicles are representative of their profusion in the natural biofilm. In areas of biofilms grown on a plastic surface, in which the cells are less tightly packed (Figure 1b), we can see that the vesicles are formed at the surfaces of cells, and the details of their structure and of the manner in which they are tethered to the cell (Figures 1c and d) are especially well resolved in these electron tomograms. We have noticed that the vesicles contain protein cargo, and have proposed that the vesicle may be the vehicle by which the transfer of outer membrane proteins such as $\mathrm{Tgl}$ and $\mathrm{CglB}$, which are involved in social and adventurous motility (respectively), can occur. It has been shown that outer membrane proteins involved in the type-IV-pili-based mechanism of social motility can be passed from cells with the genomic capability of producing them to cells of mutants that lack the gene for their production without transfer of DNA/RNA-coded information (Nudleman et al., 2005).

Lotte Sogaard-Andersen's team, at the Max Plank Institute for Terrestrial Microbiology (Marburg, Germany), in a recent review (Konovalova et al., 2010) embraced our vesicle hypothesis for protein transfer between cells within a biofilm. Proteins and any cell signaling molecules that may accompany them could be packaged into vesicles and left on swarming tracks for later uptake by neighboring or trailing cells that would then participate in motile excursions or even in fruiting body formation. We note that cell-cell signaling by diffusion of signal molecules has inherent limitations in distance and in time, because of dilution of the molecule with radial diffusion, but that the vesicular mechanism has no such limitations.

Electron tomography and 3D reconstruction allow us to resolve the membranes of the vesicles and those of the cells from which they form. Electron tomography of resin-embedded biofilms, in which fixation artifacts have been minimized 

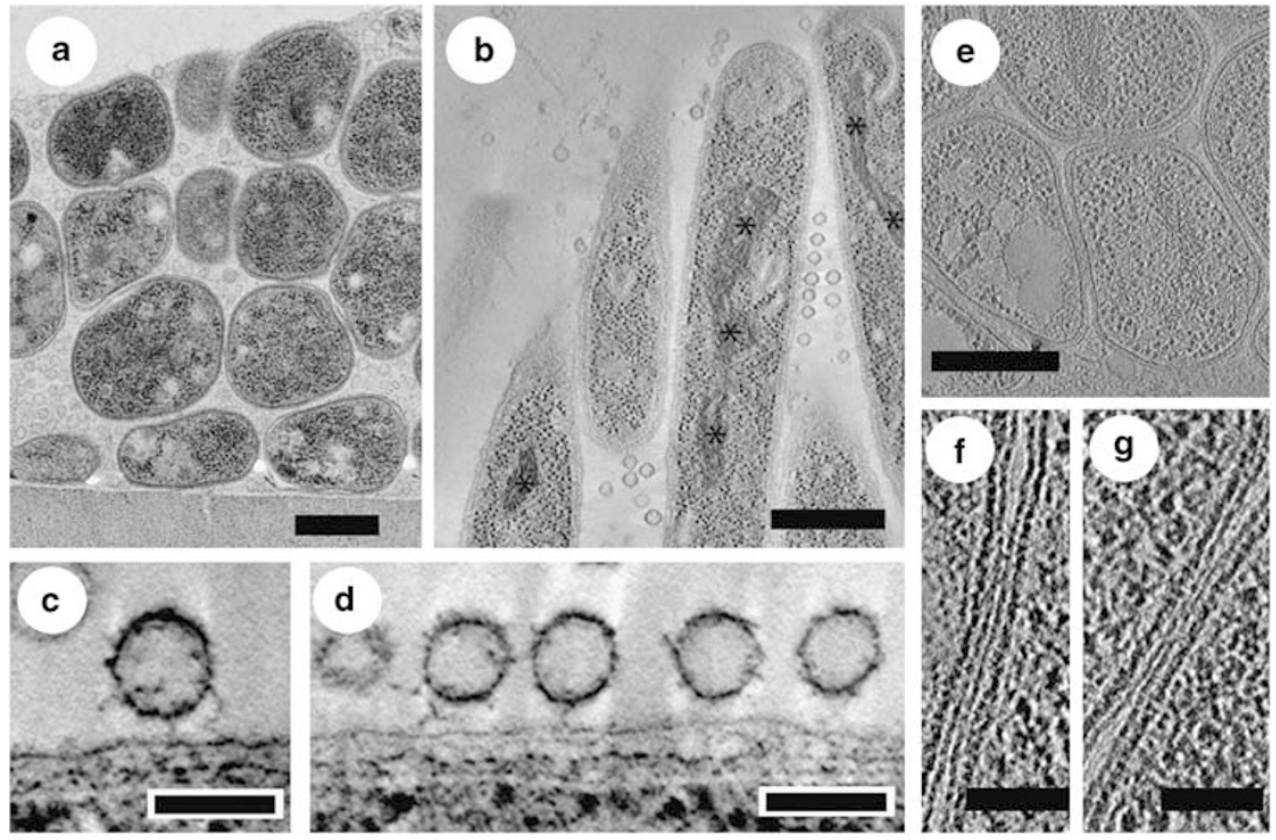

Figure 1 Ultrastructural characterization of vesicles and cell-cell contacts in high-pressure frozen and freeze-substituted Myxococcus xanthus biofilms. (a) A two-dimensional projection view image of a 100-nm thin cross-section through a thin, tightly packed biofilm resting on a cellulose substrate. Notice how bacteria accommodate each other by adjusting their shape to allow optimal dense packing. Scale bar $=500 \mathrm{~nm}$. (b) A 3-nm thin slice through a tomogram of a thick sparsely populated biofilm grown on an Isopore filter (Millipore, Billerica, MA, USA), showing regularly spaced vesicles near the bacterial surface. Scale bar $=500 \mathrm{~nm}$. (c, d) Higher magnification views of tomogram slices revealing tethering of the vesicles to the bacterial surface and each other. Notice the density inside the vesicles that may represent either protein cargo or an underlying scaffold for vesicle organization. Scale bar $=100 \mathrm{~nm}$. (e) A 3 -nm thin slice of tomogram of cross-sectioned bacteria, showing the close cell-to-cell contact. Notice also the level of detail accomplished including intracellular ribosomes and filamentous appearance of DNA material. Scale bar $=500 \mathrm{~nm}$. (f, g) Higher magnification view of cell-cell contacts showing direct outer membrane contact, but no membrane fusion. Scale bar $=100 \mathrm{~nm}$.

through high-pressure ultrarapid freezing approaches, also allows us to resolve the structures of bacterial cell outer membranes that are very closely apposed to each other in areas (Figures 1e-g). The data show that the envelope structures of neighboring cells in biofilms can be very closely apposed, with little or no intervening material mediating the contact between cells in $M$. xanthus biofilms of high density, but we have not observed fusion of outer membranes as was hypothesized by Kaiser and colleagues (Nudleman et al., 2005).

Dr Sogaard-Andersen makes a suggestion that the surfaces of $M$. xanthus cells may contain truncated assemblies of FrzCD proteins that can 'sense' the differences between the surfaces of adjacent Myxococci and inert surfaces. These sensory assemblies could only operate in regions in which the cells are in very close contact, without extensive exopolysaccharide between adjacent cells, and the appositions we observed between tightly packed cells (Figures 1e-g) could represent such proposed intimate contacts.

These paired observations, in which molecular data are placed in a structural context, are a powerful tool in the examination of intact microbial communities. We submit that this revelation that cells of $M$. xanthus may exchange proteins, by means of vesicles and/or intimate cell-cell contacts, represents another layer of complexity in the continuing story of the biofilms that predominate in natural ecosystems. Furthermore, the notion that individual cells in a biofilm community can exchange proteins, and thus endow their neighbors with characteristics (for example, specific forms of motility) for which they lack the genomic capability, represents a new level of sophistication in biofilm communities.

Further ultrastructural analysis of more mature biofilms revealed a high density of membrane structures (see Figure 2) that have the potential to connect bacteria within a biofilm and allow them to share energy and substrate and signaling molecules. With conventional sectioning and transmission electron microscopy imaging approaches, it is nearly impossible to follow such long membrane structures in a biofilm from one cell to another. We therefore have begun to use a brand new imaging approach, known as focused ion beam scanning electron microscopy, which allows the study of such mature dense biofilm communities in depth of several microns. We anticipate that this novel 3D imaging approach will yield macromolecular-resolution insight into community organization and allow visualization of its distinguished intracellular and extracellular macromolecular machines inventory. We believe that a combination of in-depth highresolution ultrastructural 3D analysis, genetics, biochemistry and correlative physiological assays are needed to understand and possibly model the coordinated, tissue-like behavior of bacteria in 


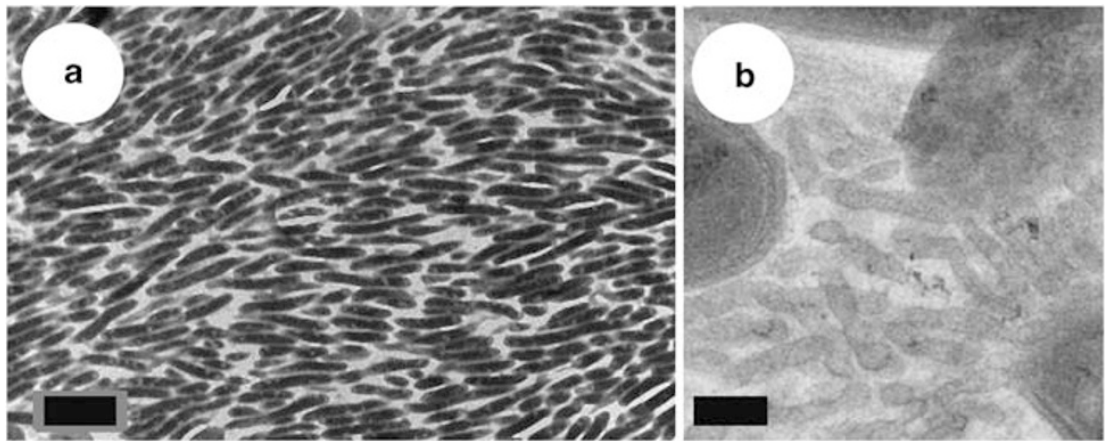

Figure 2 Potential cell-cell connections in densely populated mature Myxococcus Xanthus biofilms. (a) Low-magnification longitudinal view of $\sim 100 \mathrm{~nm}$ thin section of $M$. xanthus biofilm. Notice the preferential relative orientation of $M$. xanthus cells. Scale bar $=2 \mu \mathrm{m}$. (b) Higher magnification view into extracellular space, showing membrane structures that may connect neighboring cells. Scale bar $=100 \mathrm{~nm}$.

biofilms as a collective organism rather than individual independently acting cells.

JP Remis is at Graduate Group in Comparative Biochemistry, University of California at Berkeley, Berkeley, CA, USA and

Life Sciences Division, Lawrence Berkeley Laboratories, Berkeley, CA, USA; $J W$ Costerton is at Center for Genomic Sciences, Allegheny-Singer Research Institute, Pittsburgh, PA,

USA and

$M$ Auer is at Life Sciences Division, Lawrence Berkeley Laboratories, Berkeley, CA, USA E-mail: MAuer@lbl.gov

\section{References}

Davies DG, Parsek MR, Pearson JP, Iglewski BH, Costerton JW, Greenberg EP. (1998). The involvement of cell-tocell signals in the development of a bacterial biofilm. Science 280: 295-298.

Fuqua WC, Greenberg EP. (2002). Listening in on bacteria: acyl-homoserine lactone signaling. Nat Rev Mol Cell Biol 3: 685-695.
Konovalova A, Petters T, Sogaard-Andersen L. (2010). Extracellular biology of Myxococcus Xanthus. FEMS Microbiol Rev 34: 89-106.

Mashburn LM, Whiteley M. (2005). Membrane vesicles traffic signals and facilitate group activities in a prokaryote. Nature 437: 422-425.

McDonald KL, Auer M. (2006). High-pressure freezing, cellular tomography, and structural cell biology. Biotechniques 41: 137-141.

Nudleman E, Wall D, Kaiser D. (2005). Cell-to-cell transfer of bacterial outer membrane lipoproteins. Science 309: 125-127.

Palsdottir H, Remis JP, Schaudinn C, O’Toole E, Lux R, Shi W et al. (2009). Three-dimensional macromolecular organization of cryofixed Myxococcus Xanthus biofilms revealed by electron microscopic tomography. J Bacteriol 191: 2077-2082.

Schooling SR, Beveridge TJ. (2006). Membrane vesicles: an overlooked component of the matrices of biofilms. J Bacteriol 188: 5945-5947.

Schooling SR, Hubley A, Beveridge TJ. (2009). The interaction of DNA with biofilm-derived membrane vesicles. J Bacteriol 191: 4097-4102.

Stoodley P, Sauer K, Davies DG, Costerton JW. (2002). Biofilms as complex differentiated communities. Annu Rev Microbiol 56: 187-209.

\title{
Microbial ecosystem responses to rapid climate change in the Arctic
}

\author{
Warwick F Vincent
}

The ISME Journal (2010) 4, 1087-1090; doi:10.1038/ ismej.2010.108; published online 15 July 2010

Rapid climate change in the Arctic has begun to affect the ecology of plants and animals throughout the region, with impacts on species ranges, population dynamics and food web interactions (Grebmeier et al., 2006; Post et al., 2009). By comparison, little attention has been given to the impacts on Arctic microbial communities, despite the major and often dominant contribution of microbes to total ecosystem biomass, biodiversity, 